\title{
HIGH-SPEED POSITION DETECTOR USING NEW ROW-PARALLEL ARCHITECTURE FOR FAST COLLISION PREVENTION SYSTEM
}

\author{
Y. Oike $e^{\dagger}$, M. Ikeda $a^{\dagger \ddagger}$, and K. Asada ${ }^{\dagger \dagger}$ \\ $\dagger$ Dept. of Electronic Engineering, University of Tokyo \\ $\ddagger$ VLSI Design and Education Center, University of Tokyo \\ 7-3-1 Hongo, Bunkyo-ku, Tokyo 113-8656, Japan
}

\begin{abstract}
A high-speed position detector has wide variety of application fields such as real-time range finding and high-speed visual feedback in robot vision. In this paper, a row-parallel sensor architecture for high-speed position detection is presented. The edge of activated pixels is quickly detected by a row-parallel search circuit and its encoding cycles of $\mathrm{N}$ pixel horizontal resolution are $\mathrm{O}(\log \mathrm{N})$. The architecture keeps high-speed position detection in high pixel resolution. We have designed and fabricated the prototype position detector with a $128 \times 16$ pixel array in $0.35 \mu \mathrm{m}$ CMOS process. The measurement results show it achieves high-speed detection of $450 \mathrm{~ns}$. The high-speed position detection of the scanning sheet beam is demonstrated.
\end{abstract}

\section{INTRODUCTION}

A high-speed smart position sensor has wide variety of application fields such as real-time range finding, high-speed visual feedback in robot vision and so on. These applications require much higher speed of frame rate than the conventional imagers using serial readout and transmission. Therefore some smart sensors were proposed for high-speed position detection [1]-[6].

Fig.1 shows a fast collision prevention system with simple calculation, which is one of applications to require highspeed position detection. The scanning sheet beam activates pixels from the right to the left on the sensor plane. Two position detectors detect the edge of the activated pixels. The difference between $x_{R}$ and $x_{L}$ means the distance from the position detectors when the edge address of the left position detector is $x_{L}$ and that of the right one is $x_{R}$. High-speed position detection realizes a fast and high-resolution collision prevention system. One forward scan with N-pixel horizontal resolution requires $\mathrm{N}$ frames of the scanning sheet beam. For example, $30 \mathrm{k}$ fps is required for real-time range finding with 1k-pixel horizontal resolution. The smart sensors [3][6] are useful for applications of high-speed position detection. These frame rates, however, are not enough to realize real-time or more high-speed range finding with high pixel resolution.

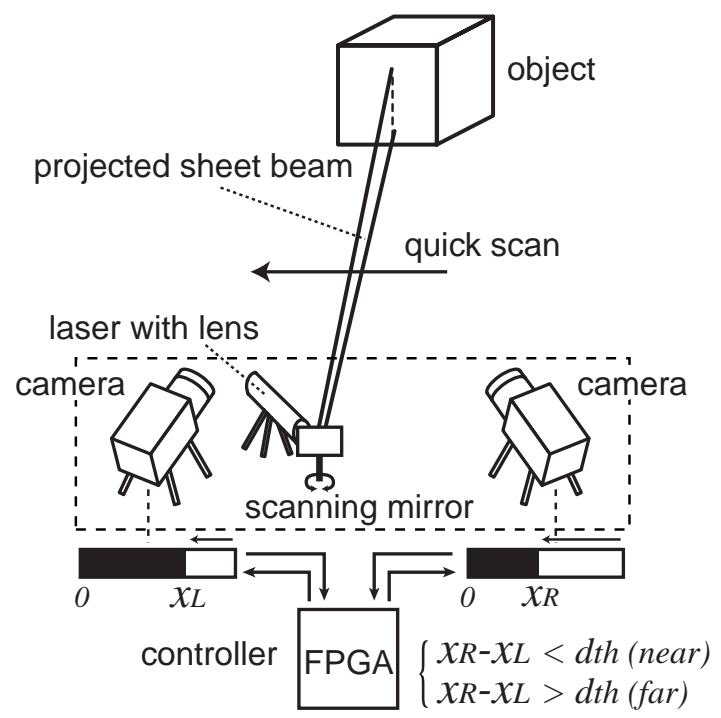

Figure 1: Fast Collision Prevention System.

In this paper, a row-parallel sensor architecture for highspeed position detection is presented. It achieves enough speed for real-time or more high-speed range finding with high pixel resolution. In this architecture, the edge of the activated pixels is quickly detected by a row-parallel search circuit and its encoding cycles of N-pixel horizontal resolution are $\mathrm{O}(\log \mathrm{N})$. We have designed and successfully tested the prototype position detector with a $128 \times 16$ pixel array in $0.35 \mu \mathrm{m}$ CMOS process.

\section{ROW-PARALLEL ARCHITECTURE}

In the position detection of a projected sheet beam, a sensor recognizes the pixels with strong incident intensity as the history of the scanning sheet beam as shown in Fig.2. Therefore it is important to quickly detect the position of the activated pixels in each row. The frontier line of the projected light provides enough information for triangulationbased range finding. Our architecture has a row-parallel search circuit for the edges of the activated pixels in each row, a row-parallel address encoder of $\mathrm{O}(\log \mathrm{N})$ and a rowparallel processor to reduce data transmission. 


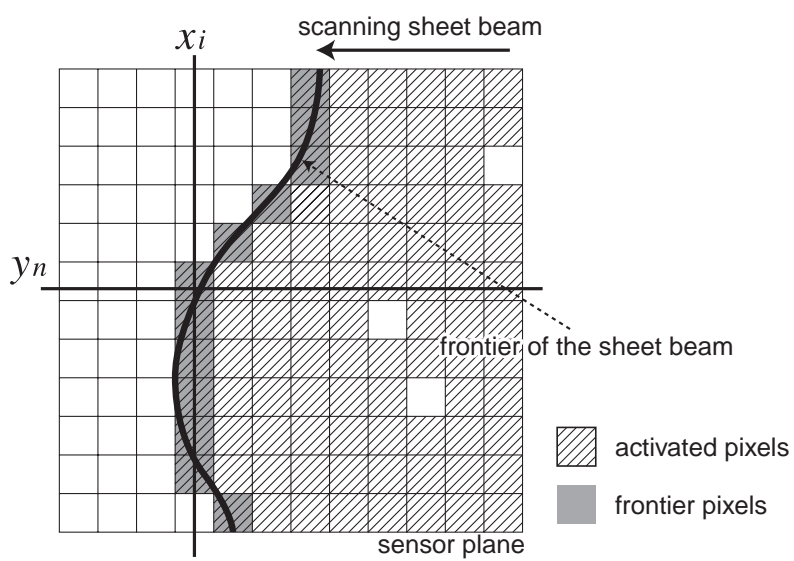

Figure 2: Captured image example of a sheet beam.

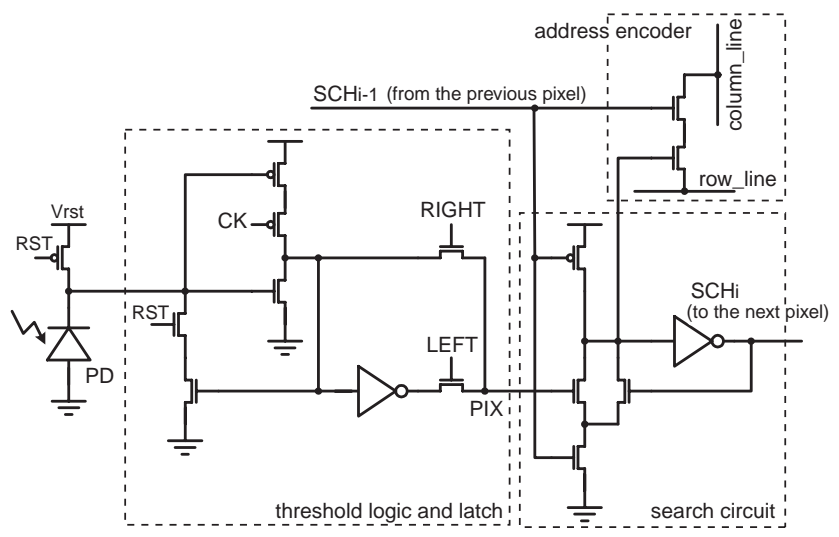

Figure 3: Pixel circuit.

\subsection{Row-Parallel Search Circuit}

Fig. 3 shows a schematic of a pixel. It has a photodiode with a reset transistor, a threshold logic and latch circuit, a search circuit, and an address encoder. The latch circuit has an XOR circuit and can invert a pixel value PIX. At the search circuit, the search signal $S \mathrm{CH}_{i-1}$ from the previous pixel passes to the next pixel when the pixel value $P I X$ is ' 1 '. On the other hand, it stops when the pixel value PIX is ' 0 '. Fig. 4 shows a procedure of the row-parallel position search. Some pixels are activated by a strong incident light and they have a pixel value PIX of ' 0 ' as shown in Fig.4(a). The search signal $S \mathrm{CH}_{0}$ is inputted to each row. It passes to the next pixel when the pixel value PIX is ' 1 '. Thus the search signal stops at the left edge $x_{i}$ of the activated pixels as shown in Fig.4(b). After a row-parallel encoding mentioned later, the pixel values PIX are inverted and the search signal starts again. It stops again at the next of the right edge $x_{j}$. The positions of the second and more activated pixels are detectable by the iteration of PIX inversions. It means that it is applicable to applications with a complex-shaped target object and/or multiple projected lights. (a) acquire image

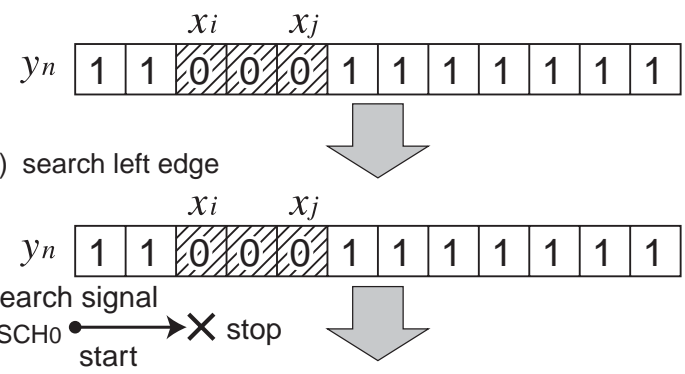

(c) invert all pixel results and search right edge

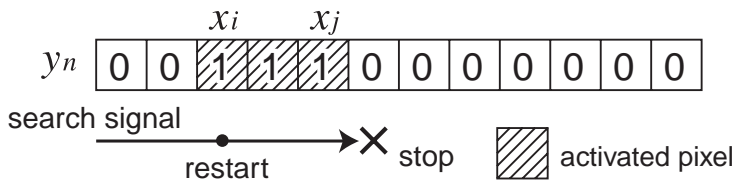

Figure 4: Procedure of row-parallel position search.

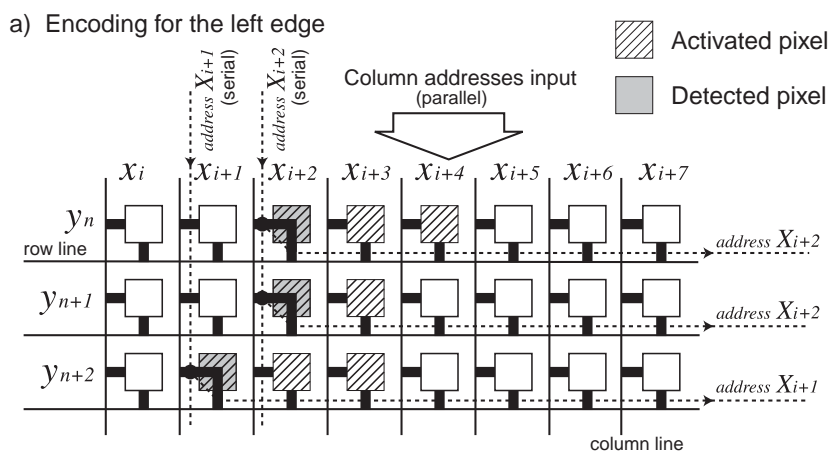

b) Encoding for the right edge

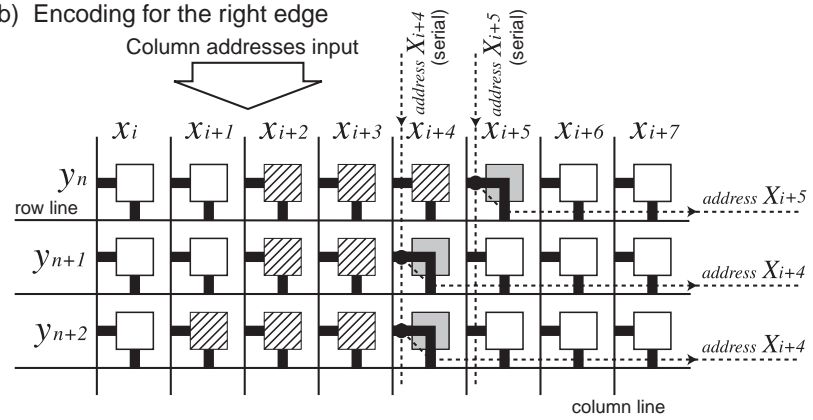

Figure 5: Method of row-parallel address encoding.

\subsection{Row-Parallel Address Encoding}

The address encoder of the pixel circuit consists of only 2 pass transistors as shown in Fig.3. At the detected pixel of each row, the column line is connected to the row line through the pass transistors as shown in Fig.5. Then, the serial-bit-streamed column address is inputted to each column line in parallel. Therefore the encoding cycles are $\mathrm{O}(\log \mathrm{N})$ at $\mathrm{N}$-pixel horizontal resolution. The compact circuit implementation and the high-speed row-parallel encoding realize high-speed position detection in high pixel resolution. 


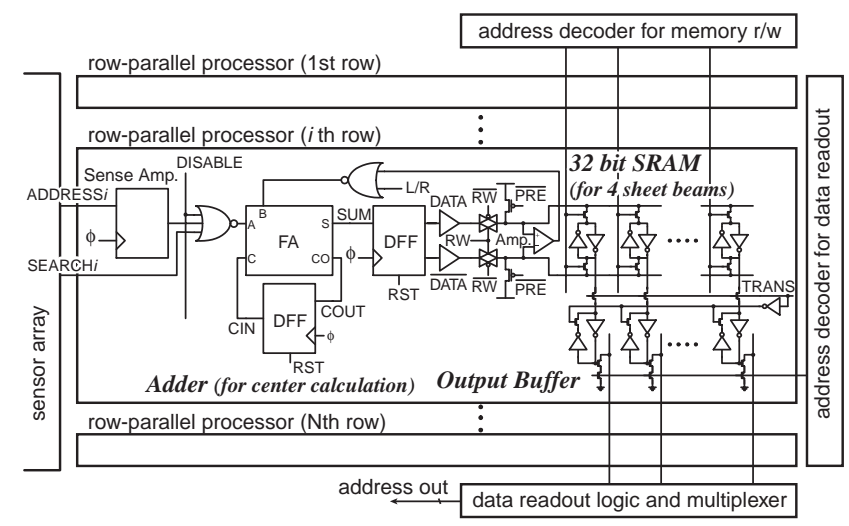

Figure 6: Structure of row-parallel processor.

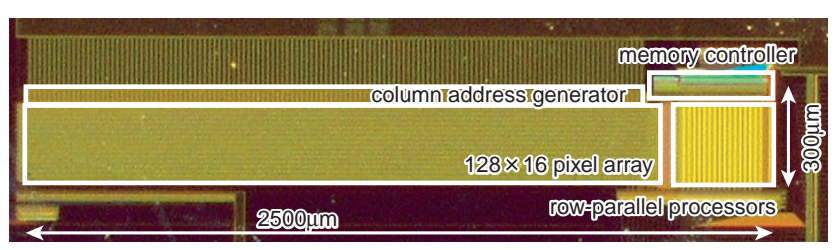

Figure 7: Chip microphotograph.

\subsection{Row-Parallel Processor}

The photo detector has a row-parallel processor as shown in Fig.6. It consists of a latch sense amplifier to get the address data, a full adder, random access memories with a read/write circuit, output buffers for pipe-lined data readout, and some control logics. The row-parallel address encoding can acquire the addresses of $x_{i}$ and $x_{j}+1$ when the edges of the activated pixels are $x_{i}$ and $x_{j}$. The processor calculates the center position of the detected pixels and reduces data transmission. And also it realizes to get the positions of multiple sheet beams in one frame. The row-parallel processor can be extended to deal with another data processing. For example, multiple samplings per frame can be realized for high sub-pixel accuracy when a timing memory and its control logics are implemented.

\section{CHIP IMPLEMENTATION}

We designed and fabricated a prototype position detector in $0.35 \mu \mathrm{m}$ CMOS process ${ }^{1}$. Fig. 7 shows a microphotograph of the fabricated chip. It consists of a $128 \times 16$ pixel array, a column address generator, row-parallel processors with 32 bit SRAM per row and a memory controller. The pixel circuit has 1 photo diode and 18 transistors in $16.25 \mu \mathrm{m} \times$ $16.25 \mu \mathrm{m}$ pixel area with $20.15 \%$ fill factor.

\footnotetext{
${ }^{1}$ The VLSI chip in this study has been fabricated through VLSI Design and Education Center (VDEC) in collaboration with Rohm Corp. and Toppan Printing Corp.
}

Table 1: Specifications of the prototype chip.

\begin{tabular}{ll}
\hline Process & $0.35 \mu \mathrm{m}$ CMOS 3-metal 1-poly-Si \\
Sensor size & $2.5 \mathrm{~mm} \times 0.3 \mathrm{~mm}$ \\
\# pixels & $128 \times 16$ pixels \\
Pixel size & $16.25 \mu \mathrm{m} \times 16.25 \mu \mathrm{m}$ \\
\# trans. / pixel & 18 transistors \\
Fill factor & $20.15 \%$ \\
\hline
\end{tabular}

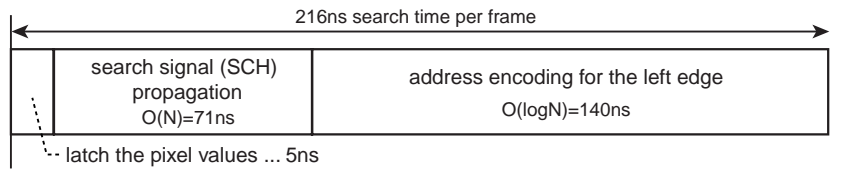

Figure 8: Simulated search time per frame for position detection of the fabricated chip.

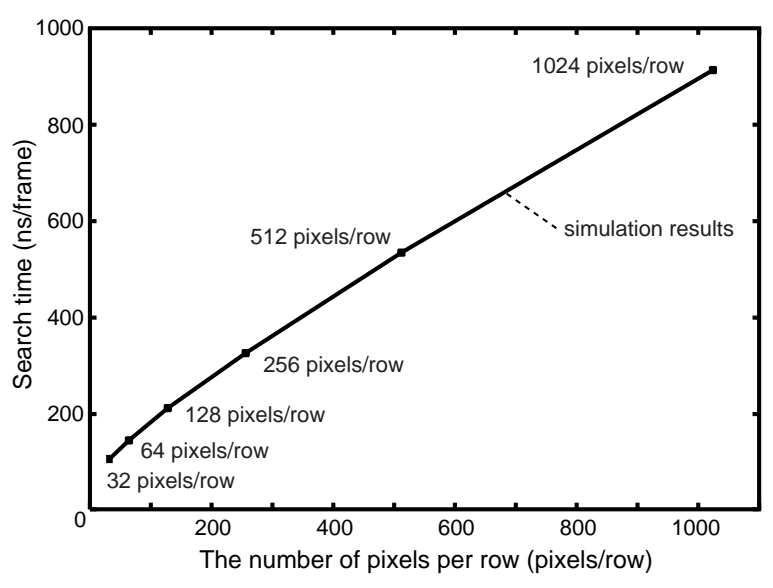

Figure 9: Search time in high pixel resolution.

\section{PERFORMANCE EVALUATION}

Fig. 8 shows search time per frame for position detection simulated by HSPICE. The maximum propagation delay of the search signal is $71 \mathrm{~ns}$ and the 7-bit address encoding for 128 columns takes $140 \mathrm{~ns}$. The total search time to get the position of the left edge of the activated pixels is 216 ns per frame. In a single sampling per frame, the frame interval is a total of the integration time of incident light and the search time for the left and right edges. The frame interval, however, is only the search time for the left edge in multi samplings per frame. That is, the sensor detects the frontier positions of the scanning sheet beam at each time during the integration of incident light. The present architecture achieves $918 \mathrm{~ns}$ search time per frame at 1024-pixel horizontal resolution. Generally real-time range finding of $1024 \times 1024$ pixels requires $32.5 \mu$ s search time per frame. The present architecture realizes enough speed not only for real-time range finding but also for beyond-real-time range finding and visual feedback. 
Table 2: Performance comparisons.

\begin{tabular}{l||c|c}
\hline & frame rate & \# pixels \\
\hline Our detector (single-sampling) & $32.2 \mathrm{k} \mathrm{fps}$ & $128 \times 16$ \\
& $(31.4 \mathrm{k} \mathrm{fps})$ & $1024 \times 1024$ \\
Our detector (multi-sampling) & $2.22 \mathrm{M} \mathrm{fps}$ & $128 \times 16$ \\
& $(1.09 \mathrm{M} \mathrm{fps})$ & $1024 \times 1024$ \\
\hline Brajovic et al. [4] & $6.4 \mathrm{k} \mathrm{fps}$ & $32 \times 64$ \\
Sugiyama et al. [6] & $3.3 \mathrm{k} \mathrm{fps}$ & $320 \times 240$ \\
Required fps for real time & $30.7 \mathrm{k} \mathrm{fps}$ & $1024 \times 1024$ \\
\hline
\end{tabular}

Frame rates in parentheses are simulation results.

\section{MEASUREMENT RESULTS}

The measurement system of the fabricated chip has been developed as shown in Fig.1. In the system, the position detector and a scanning mirror are controlled by FPGA and the acquired position data are transferred to a PC. The FPGA was operated at $80 \mathrm{MHz}$ due to the limitation of the testing equipment. The search time was $450 \mathrm{~ns}$ per frame and the integration time of incident light was $30 \mu \mathrm{s}$ at $V_{r s t}=1.4 \mathrm{~V}$. Fig.10 shows the measurement results of the present position detector. Fig.10(a) shows the position of the left and right edges of the activated pixels. That is, the projected sheet beam is located between these edges on the sensor plane. The position detector has the processor to calculate the center position on the chip. Only the center address can be acquired to reduce data transmission as shown in Fig.10(b). Fig.10(b) shows sequentially captured positions of the scanning sheet beam of $2 \mathrm{kHz}$ by single sampling per frame. It takes $30.9 \mu$ s per frame. It has 256 sub-pixel resolution due to the center calculation. Fig.10(c) shows the frontier positions of the scanning sheet beam. It takes 0.45 $\mu$ s per frame. The results show the frame rate is $32.2 \mathrm{k}$ fps and $2.22 \mathrm{M}$ fps in a single sampling and multi samplings per frame respectively. The performance comparisons are shown in Table 2.

\section{CONCLUSIONS}

A row-parallel sensor architecture for high-speed position detection has been proposed. It has been designed and fabricated in $0.35 \mu \mathrm{m}$ CMOS process and successfully tested. The prototype position detector has $128 \times 16$ pixels and it achieves $450 \mathrm{~ns}$ search time per frame. In the measurement system using multi samplings per frame, the high-speed position detection of a scanning sheet beam is realized at $2.22 \mathrm{M}$ fps. It is enough speed not only for real-time range finding but also for beyond-real-time range finding and visual feedback such as a fast collision prevention system. We have also shown its applicability to higher pixel resolution such as $1024 \times 1024$ pixels. (a) result of the sheet beam detection

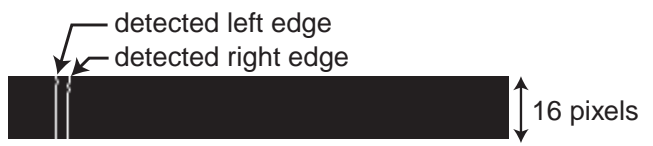

(b) single sampling per frame

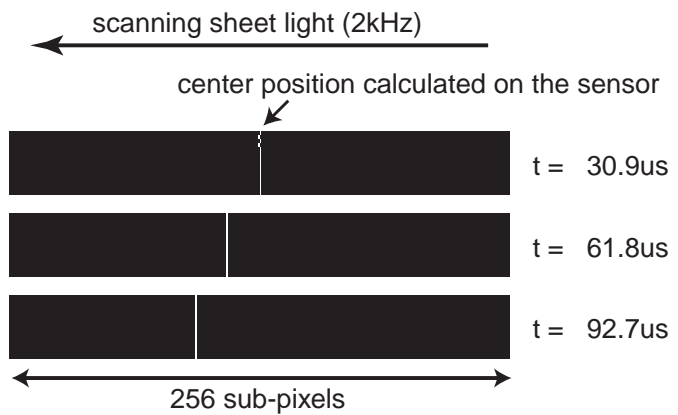

(c) multi samplings per frame

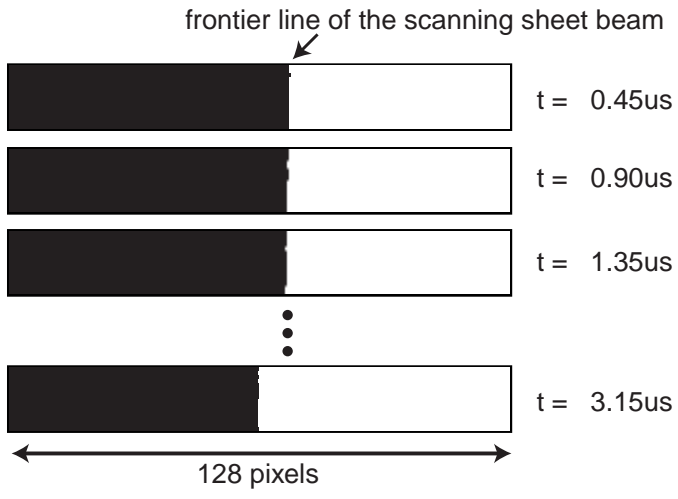

Figure 10: Measurement results.

\section{REFERENCES}

[1] V. Brajovic and T. Kanade, "Computational Sensor for Visual Tracking with Attention," IEEE Journal of Solid-State Circuit, vol. 33, pp. 1199 - 1207, 1998.

[2] T. Nezuka, M. Hoshino, M. Ikeda and K. Asada, "A Position Detection Sensor for 3-D Measurement," in Proc. European Solid-State Circuits Conference, pp. 412 - 415, 2000.

[3] M. de Bakker, P. W. Verbeek, E. Nieuwkoop and G. K. Steenvoorden, "A Smart Range Image Sensor," in Proc. European Solid-State Circuits Conference, pp. 208 - 211, 1998.

[4] V. Brajovic, K. Mori and N. Jankovic, "100 frames/s CMOS Range Image Sensor,” ISSCC Dig. of Tech. Papers, pp. 256 $-257,2001$.

[5] S. Yoshimura, T. Sugiyama, K. Yonemoto and K. Ueda, "A 48k frame/s CMOS Image Sensor for Real-time 3-D Sensing and Motion Detection," ISSCC Dig. of Tech. Papers, pp. 94 $-95,2001$.

[6] T. Sugiyama, S. Yoshimura, R. Suzuki and H. Sumi, "A 1/4inch QVGA Color Imaging and 3-D Sensing CMOS Sensor with Analog Frame Memory," ISSCC Dig. of Tech. Papers, pp. $434-435,2002$. 